\title{
Laboratory evidence of slow-cooling for carbon droplets in red-giant atmospheres
}

\author{
Melanie Lipp ${ }^{1}$, Taylor J. Savage ${ }^{1}$, David Osborn ${ }^{1}$ and P. Fraundorf ${ }^{1,2}$ \\ ${ }^{1}$ Physics \& Astronomy and Center for Nanoscience, U. Missouri StL, USA \\ ${ }^{2}$ Physics Department, Washington University, St. Louis, MO, USA
}

Micron-sized presolar carbon spheres condensed before the origin of our solar system, in the atmosphere of asymptotic giant branch stars from carbon atoms dredged up following nucleosynthesis in the stellar interior, have been extracted by dissolution from primitive carbonaceous chondrite meteorites, identified by noble-gas and major-element isotopic analysis, and available to electron microscopists on earth for over two decades [1-4]. Some of these spheres appear to consist of graphite condensed on carbide grains, but others instead show "graphite-onion" rims that surround a spherical-core of unlayeredgraphene i.e. carbon that in diffraction shows graphite (hk0) spacings only. The high spatial-frequency tail of these graphene lines [5] suggests a graphene-sheet coherence width in the 4-nm size range [1], consistent with electron-phase-contrast images of single (sometimes segmented) edge-on sheets $[2,4]$.

Carbon-vapor cooling below $3915 \mathrm{~K}$ at low pressure in a containerless setting is expected [6] (and seen experimentally [7]) to condense as a supercooled liquid. Like other metallic liquids [8] this may not solidify until perhaps $30 \%$ below the (in graphite's case virtual) melting temperature, i.e. around $0.7 \times 4600 \mathrm{~K} \approx 3220 \mathrm{~K}\left(2950^{\circ} \mathrm{C}\right)$. Simple model calculations suggest that a half-micron diameter droplet of liquid carbon at $3915 \mathrm{~K}$, injected into a "cool" red giant photosphere, will take tens of microseconds to radiatively cool below $3220 \mathrm{~K}$. The cooling time for such carbon droplets in a stellar atmosphere may be orders of magnitude longer.

Molecular dynamic calculations of carbon solidification generally take place over much shorter (i.e. picosecond) time scales [9]. Significant graphitization can nonetheless be achieved [10], perhaps because the simulated anneal can be done at temperatures just below melting. Because these calculations can be done with tens of thousands of atoms, we've put resulting atom position lists into our on-line strong-phase-object TEM simulator [11] for comparison to model as well as experimental [2] presolar onion-core images.

The differences are that in experimental images of presolar onion cores, (a) the coherence widths of graphene sheets are much larger (approaching $4[\mathrm{~nm}]$ ) than in the simulations, (b) where seen edge on graphene sheets tend to be planar rather than curved (oft-times intersecting another straight-line segment at a point), and (c) unlike many of the simulations we don't see signs of parallel-layer formation. All of these features may be consistent with a much longer and cooler crystallization period than possible today with a simulated anneal.

Longer crystallization periods may, however, be possible to investigate via the laboratory synthesis of such material. In particular, an evaporating oven strategy [6] (cf. Fig. 2) may be modified to adjust the cooling rate for micron sized carbon onions created therein. The radiative steady-state may be controlled by adjusting the temperature of surfaces "visible" to a condensed particle. The "fall time" for such particles, before they hit a cooler container wall, may require control of geometric (gravity and inertial) forces in the oven. However in earth's surface gravity fall times in vacuo over a millimeter of distance are over 10 milliseconds, already a factor of 1000 longer than the minimum radiative cooling time for a 
condensed particle, and a billion times longer than some molecular dynamics simulation runs.

References:

[1] Thomas J. Bernatowicz et al. (1996), Astrophysical Journal 472, 760.

[2] P. Fraundorf and Martin Wackenhut (2002) Ap. J. Lett. 578(2) L153-156.

[3] Thomas K. Croat et al. (2005) Astrophysical Journal 631, 976-987.

[4] Eric Mandell (2007) Ph.D. Dissertation at UM-St Louis/Rolla.

[5] B. E. Warren (1941) "X-ray diffraction in random layer lattices", Phys. Rev. 59(9), 693.

[6] P. Fraundorf et al. (2016) Microscopy and MicroAnalysis 22:S3, 1816-1817 HAL-01356394.

[7] W.A. De Heer et al. (2005) Science 307:5711, pp. 907-910.

[8] K. F. Kelton and A. L. Greer (2010) Nucleation in Condensed Matter (Elsevier, Amsterdam).

[9] T. D. Nguyen (2017) Computer Physics Communications 212, 113.

[10] Carla de Tomas et al. (2016) Carbon 109, 681-693, http://www.carbonpotentials.org/.

[11] P. Fraundorf et. al. (2016) Microscopy and Microanalysis 22:S3, 1442-1443 HAL-01362470.

Fig. 1: Strong-phase object HRTEM images of de Tomas [8] annealed carbon clusters, discussed in text.

\begin{tabular}{|c|c|c|c|}
\hline density & Tersoff & REBO & EDIP \\
\hline \multirow{3}{*}{$2[\mathrm{~g} / \mathrm{cc}]$} & 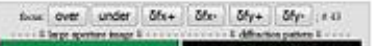 & 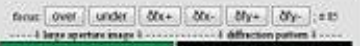 & 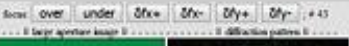 \\
\hline &  & 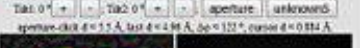 & 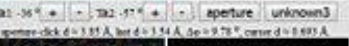 \\
\hline & 号 & 口 & 5 \\
\hline
\end{tabular}

Figure 1. Graphitized carbon clusters with 17000 atoms from [8], as imaged in our on-line JS/HTML5 simulator at sites.google.com/site/electrondetectives.
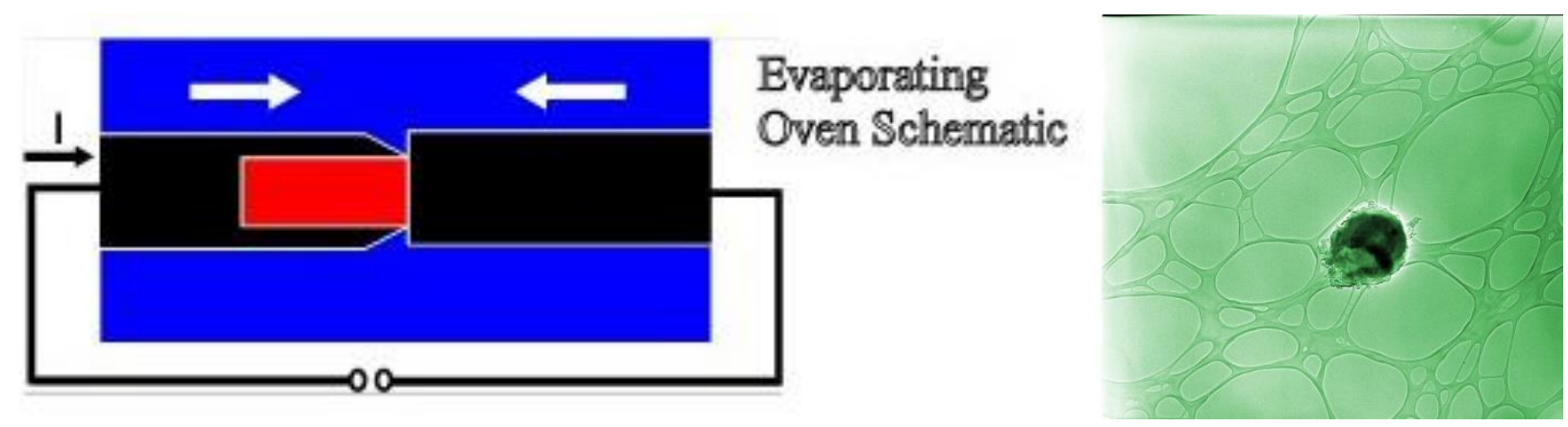

Figure 2. Left: Resistively-heated oven design; Right: Micron-sized graphite onion condensed therein. 